\title{
Progress on the Thompson-Lampard calculable capacitor project at BIPM
}

\author{
P. Gournay ${ }^{a}$, N. Fletcher, L. Robertsson, M. Stock \\ Bureau International des Poids et Mesures (BIPM), Pavillon de Breteuil, 92312 Sèvres Cedex, France
}

\begin{abstract}
Résumé. Nous présentons dans le présent document une vue d'ensemble du projet de détermination directe de la constante de von Klitzing $R_{\mathrm{K}}$, en cours de réalisation au Bureau International de Poids et Mesures (BIPM). Cette détermination est effectuée dans le contexte général de la révision du Système International d'unités qui sera effective à partir de 2018. L'objectif visé est de mesurer avec une incertitude relative réduite à $1.10^{-8}$. Pour cela un nouveau condensateur calculable a été réalisé et une chaine de mesure dédiée implémentée.
\end{abstract}

\section{Introduction}

Within the context of the forthcoming redefinition of the International System of units (SI), expected for 2018, electrical units will be based on two fundamental constants: $h$ the Planck constant and $e$ the elementary charge of an electron [1].

As a result, the quantum Hall and the Josephson effects will definitely become the natural paths for the mise en pratique of the impedance and voltage units, the ohm and volt respectively. Even if these effects are currently widely used in National Metrology Institutes (NMIs) due to their very good reproducibility, they do not allow generating these units in terms of SI base units with an uncertainty better than about 1 part in $10^{7}$ in relative.

Indeed, these two quantum effects allow to produce quantized values of resistance and voltage directly linked to two phenomenological constants, $R_{\mathrm{K}}$ and $K_{\mathrm{J}}$, respectively the von Klitzing and Josephson constants. Physical theories describing these effects allow to demonstrate that they depend only on $h$ and $e$ through the relations $R_{\mathrm{K}}=h / e^{2}$ and $K_{\mathrm{J}}=2 e / h$. At present, experimental tests of these relations are ongoing and electrical metrology instead relies on conventional agreed values for $R_{\mathrm{K}}$ and $K_{\mathrm{J}}$. As a result, the SI values of the quantized voltages and resistances generated through the quantum Hall and Josephson effects have minimum relative uncertainty equal to $1 \times 10^{-7}$ and $4 \times 10^{-7}$ respectively.

In order to improve this situation and before to proceed to the re-definition of the SI, the Conférence Générale des Poids et Mesures (CGPM) and the Comité International des Poids et Mesures (CIPM) have for many years encouraged NMIs and BIPM to implement experimental setups to perform the measurement of the relevant fundamental constants, including $R_{\mathrm{K}}$ and $K_{\mathrm{J}}$, with reduced uncertainties to a level of the order of $10^{-8}$ in relative. Several scientific institutes including the BIPM have started the development of calculable capacitors [2] and watt balances [3] allowing the determination with improved uncertainties of $R_{\mathrm{K}}$ and $K_{\mathrm{J}}$ respectively (note that watt balances measure the product $K_{\mathrm{J}}^{2} R_{\mathrm{K}}$ allowing to derive the SI value of $h$ considering the above relations).

In the following we present a brief overview of the ongoing project of measurement of $R_{\mathrm{K}}$ at BIPM with a target uncertainty of the order of 1 part in $10^{8}$. A major step for this project to succeed is the construction of a calculable capacitor allowing the generation of repeatable capacitance variations with an uncertainty of few a parts in $10^{9}$. Nevertheless, the associated measuring chain allowing linking those capacitance variations to the quantum resistance obtained from the quantum Hall effect must of course also not introduce measuring uncertainties larger than few parts in $10^{9}$.

\section{Principle of determination of $\boldsymbol{R}_{\mathrm{K}}$}

The principle of measurement of $R_{\mathrm{K}}$ in the SI, schematized in figure 1, consists in linking the farad, realized by a calculable capacitor, to the ohm obtained through the quantum Hall effect. Starting from the calculable capacitor, the measuring procedure consists in transferring the calculable capacitance variation it generates $(0.4 \mathrm{pF})$ to a $1 \mathrm{pF}$ standard using a $2.5: 1$ twoterminal coaxial impedance bridge. This transfer is carried out at five frequency values $513 \mathrm{~Hz}, 1027 \mathrm{~Hz}$, $1541 \mathrm{~Hz}, 3082 \mathrm{~Hz}$ and $6184 \mathrm{~Hz}$ [4].

Then, a second 10:1 two-terminal pair coaxial impedance bridge is used to transfer the $1 \mathrm{pF}$ value to a pair of $1 \mathrm{nF}$, $2 \mathrm{nF}$ and $3 \mathrm{nF}$ capacitors through the use of $10 \mathrm{pF}$ and $100 \mathrm{pF}$ transfer standards. Depending on the operating frequency, either the 1,2 or $3 \mathrm{nF}$ capacitors serve to calibrate a pair of $51.6 \mathrm{k} \Omega$ ac-resistors using a multifrequency quadrature bridge [4]. The calibration of these resistors, or more specifically the product of their resistance values, is performed for the five frequency

\footnotetext{
a pierre.gournay@bipm.org
}

This is an Open Access article distributed under the terms of the Creative Commons Attribution License 4.0, which permits unrestricted use, distribution, and reproduction in any medium, provided the original work is properly cited. 
values mentioned above and the resistance value at dc is deduced from the extrapolation to zero of the frequency dependence curve. Notice that the frequency dependence of the calculable capacitor can be modelled and is consequently known, avoiding the need to correct the resistance value from their frequency dependence before extrapolation. Nevertheless, as shown on figure 1, the frequency corrections of the $51.6 \mathrm{k} \Omega$ resistors can also be measured in a two steps comparison process with a $1290.6 \Omega$ calculable Haddad resistor. This will allow a verification of the modelling of their frequency dependence based on the measurements with the calculable capacitance.

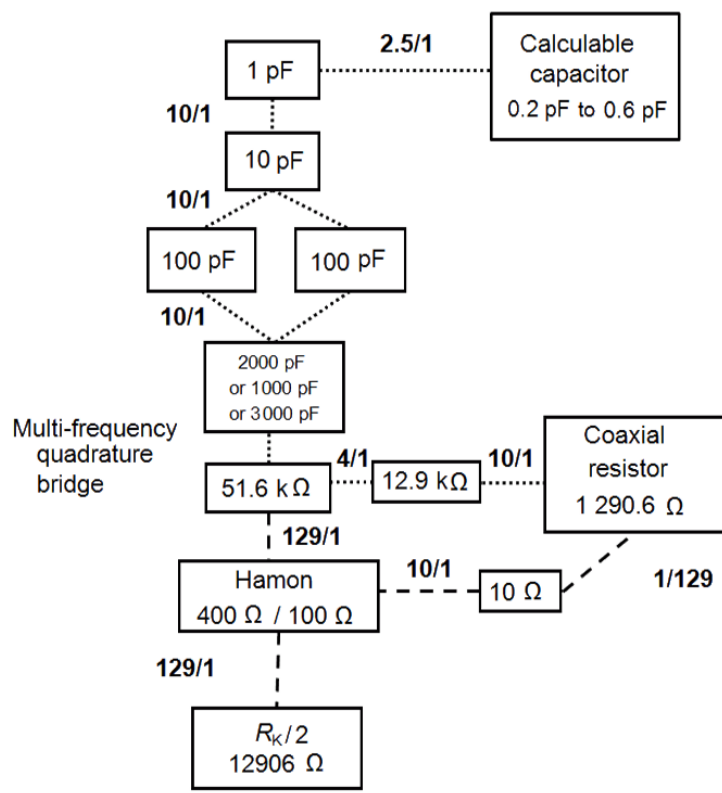

measurements in the range $500 \mathrm{~Hz}$ to $6000 \mathrm{~Hz}$ using coaxial AC bridges

_ _ . : measurements at $1 \mathrm{~Hz}$ (or at DC)

using a current comparator

Figure 1: Measuring chain used at BIPM to link the farad to the ohm

The extrapolated value at $\mathrm{dc}$ of the pair of $51.6 \mathrm{k} \Omega$ resistors can thus be compared to their dc-value calibrated with respect to the quantum Hall effect considering the conventional value of $R_{\mathrm{K}}\left(R_{\mathrm{K}-90}=\right.$ $25812.807 \Omega$ [5]). This calibration is carried out against the quantized resistance $R_{\mathrm{K}} / 2$ (filling factor $\mathrm{i}=2$ ) using a 129:1 current comparator bridge operated at $1 \mathrm{~Hz}$ [6] and through a transfer Hamon network $400 \Omega / 100 \Omega$. Notice that operating the quantum Hall effect at $1 \mathrm{~Hz}$ instead of dc implies no correction.

This comparison of dc resistance values obtained from the calculable capacitor and from the quantum Hall effect leads to the determination of the difference between the conventional value of $R_{\mathrm{K}}, R_{\mathrm{K}-90}$, and its SI value.

The whole measuring chain, including all the ac-coaxial impedance comparison bridges (transformer bridges) and the transfer standards is operational.

\section{The BIPM calculable capacitor}

As already mentioned, a major difficulty in implementing the measuring chain presented above consists in designing, assembling and aligning the calculable capacitor. This is mainly due to the fact that the targeted level of uncertainty implies that the geometry of the capacitor is defined to better than $100 \mathrm{~nm}$.

The project to build such a capacitor at BIPM has started in 2005 and has been conducted in collaboration with the National Measurement Institute of Australia (NMIA).

This new calculable capacitor is an improved version of a previous design by the CSIRO of Australia which was developed and built about 40 years ago. Its principle of functioning is based on a theorem in electrostatics described by A. Thompson and D. Lampard from CSIRO in 1956 [7].

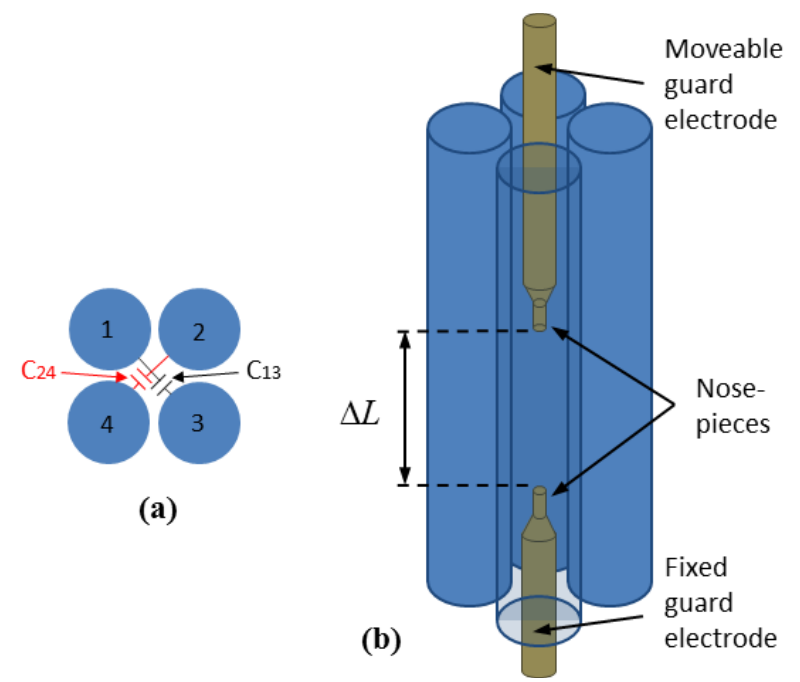

Figure 2: Practical realization of Lampard's theorem; (a) normal cross section of a four electrode bars system with cross-capacitances between opposite pairs of bars, (b) four bars arrangement with guard electrodes

A practical realization of this theorem is shown in figure 2. It consists of a cylindrical capacitor composed of four vertical electrode bars separated by small isolating gaps (negligible in theory). The cross-section of the electrode system remains invariant along the axis of the four electrode bars (meaning a perfect cylindricity of the bas and parallelism of their axis). In the inter-electrode space two guard electrodes, one fixed and the other moveable, perfectly aligned with the cylindrical axis of the capacitor cross-section define the electrical length of the capacitor. In a two-step measurement procedure, the capacitances of the opposite pairs of bars $\left(\mathrm{C}_{13}\right.$ and $\mathrm{C}_{24}$ on fig. $2 \mathrm{a}$ ) are measured before and after the position of the moveable guard electrode is changed by a distance $\Delta L$. Since the electrode arrangement is symmetrical, the two opposite cross capacitances are equal and the Lampard theorem allows the calculation of the capacitance variation corresponding to the mean of cross capacitances increases, $\Delta C$, from the simple relation,

$$
\Delta C=\varepsilon_{0}(\operatorname{Ln} 2 / \pi) \Delta L
$$


This capacitance variation $\Delta C$ is then transferred through a substitution measurement to a capacitance standard ( $1 \mathrm{pF}$ in the measuring chain of figure 1 ).

In order to reach the needed accuracy on the value of $\Delta C$ the distance $\Delta L$ is measured by interferometry and, de facto, related to the meter if the laser source used in the interferometer is servoed on a molecular absorption line ( $\mathrm{I}_{2}$ line in our experiment). This means that a calculable capacitor allows to link the farad to the meter and as a consequence that the value of $R_{\mathrm{K}}$ measured as described in paragraph 2 is linked to the meter and the second.

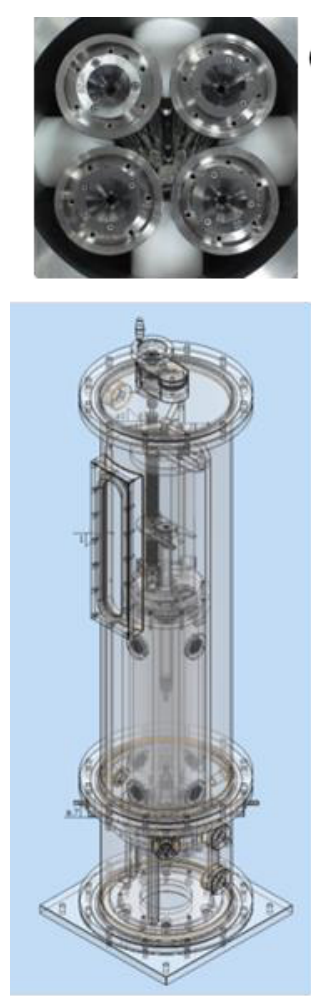

(a)

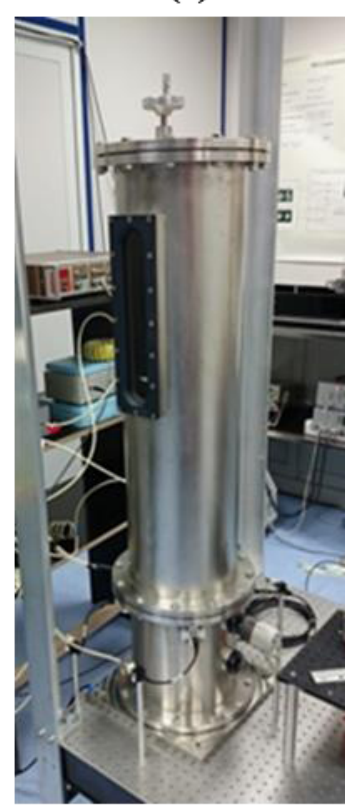

(b)

Figure 3: (a) Top view of the main electrode bars during assembly; (b) Drawing in transparence of the BIPMNMIA calculable capacitor (drawing from NMIA); (c) Picture after assembling at BIPM.

The basic design of the BIPM-NMIA calculable capacitor is as shown in figure 2. The initial requirements needed to reach the target uncertainty of 1 to few parts in $10^{9}$ on the value of $\Delta C$ as well as the final design choices are described in detail in references [2, 8-10].

It is made up of four main electrode bars, $50 \mathrm{~mm}$ in diameter and $470 \mathrm{~mm}$ length, with a cylindricity of better than $100 \mathrm{~nm}$ [2]. The main bars are installed vertically in the capacitor and aligned to be parallel with their axis standing on the edges of a square and not skewed. The alignment is realized with a dedicated set of capacitance probing tools with an accuracy equivalent to the main bars geometrical tolerance, that is $\leq 100 \mathrm{~nm}$. The moveable guard electrode is $22 \mathrm{~mm}$ in diameter (as is the fixed one) and can be moved over a distance of about $205 \mathrm{~mm}$ to generate a capacitance variation of $0.4 \mathrm{pF}$ (between 0.2 and $0.6 \mathrm{pF}$ ). The isolating gaps between each of the main electrodes and between the main electrodes and the guard electrodes are respectively of 3 $\mathrm{mm}$ and $1.5 \mathrm{~mm}$.

As shown figure 2, the guard electrode ends are equipped with semi-conical nonepieces. They allow compensating for residual misalignment of the main electrodes. The nosepieces are hollow and carry semi-transparent mirrors, forming a Fabry-Perot cavity for the interferometric measurement of the moveable guard displacement [10].

The calculable capacitor is entirely shielded and mounted in a vacuum enclosure in order to avoid measurement errors (length and capacitance), figure 3. For more information about the BIPM-NMIA calculable capacitor, the reader is invited to refer to the references noted below.

\section{Conclusion}

The BIPM-NMIA calculable capacitor is assembled and a first alignment has been realized. The measuring chain is fully operational and has been fully checked. First series of measurements have been carried out in order to identify eventual design problems.

The results obtained are quite encouraging even if small misalignment errors have been detected. These errors are understood and are mainly due to the lack of precision of one of the probing tools used in the alignment procedure. A new kind of probe is currently under construction which should allow correcting for this. Outside this problem, the accuracy of the new calculable capacitor is as good as expected from its design and puts within reach the measurement of $R_{\mathrm{K}}$ with the targeted uncertainty.

\section{References}

1. Resolutions of the $24^{\text {th }}$ and $25^{\text {th }}$ CGPM (2007 and 2014) - www.bipm.org

2. G.W. Small, IEEE Trans. Instr. and Meas., Vol. 60, $\mathrm{n}^{\circ} 7,2489,(2011)$

3. A. Eichenberger et al., Eur. Phys. J. Special Topics, 172, 363, (2009)

4. F. Delahaye and R. Goebel, IEEE Trans. Instrum. and Meas., Vol. 54, n², 533, (2005)

5. P.J. Mohr et al., Rev. Mod. Phys., 84, 1527, (2012)

6. F. Delahaye, IEEE Trans. Instrum. and Meas., Vol. 40, ${ }^{\circ} 6,883,(1991)$.

7. A.M. Thompson, D.G. Lampard, Nature, 177, 888, (1956)

8. G.W. Small, J.R. Fiander, Conference on Precision Electromagnetic Measurements Digest, 485, London, (2004)

9. J.R. Fiander, G.W. Small, Conference on Precision Electromagnetic Measurements Digest, 652, Torino, (2006)

10. N. Fletcher et al., Conference on Precision Electromagnetic Measurements Digest, 252, Washington, (2012) 